\title{
Nonstandard theta Milstein method for solving stochastic multi-strain tuberculosis model
}

\author{
Nasser H. Sweilam ${ }^{*}$ (D) and Seham M. AL-Mekhlafi ${ }^{2}$
}

\author{
*Correspondence: \\ nsweilam@sci.cu.edu.eg \\ This a joint paper all the manuscript \\ work are done in an equal \\ percentage of participation \\ 'Department of Mathematics, \\ Faculty of Science, Cairo University, \\ Giza, Egypt \\ Full list of author information is \\ available at the end of the article
}

\begin{abstract}
In this article, a novel stochastic multi-strain tuberculosis model is presented. Numerical simulations for this model are the main aim of this work. A non-standard theta Milstein method is constructed to study the proposed model, where the proposed method is based on choosing the weight factor theta. The main advantage of this method is it can be explicit or implicit with large stability regions using the idea of the weighed step introduced by R.E. Mickens. Mean-square stability of nonstandard theta Milstein method is studied. The new scheme shows a greater behavior compared to the theta Milstein method. It is concluded that the proposed scheme preserves the positivity of the solution and numerical stability in larger region than the standard method.
\end{abstract}

Keywords: A non-standard theta Milstein method, Multi-strain Tuberculosis, Mean square stability analysis

\section{Introduction}

It is well known that the solution of stochastic differential equations is either difficult in general or we do not have explicit solutions. Numerical schemes provide an easy way to integrate these equations, see [1-8]. Moreover, numerical simulations are considered the only way to solve these mathematical models in general or to derive the desired information. Therefore, the accuracy of these numerical solutions could be a major factor in choosing the appropriate numerical method and solving mathematical models. The usual numerical schemes even in the deterministic case such as Euler, Runge-Kutta, and Euler-Maruyama in the stochastic case do not preserve dynamical properties without conditions on the time step of the numerical integration; see [9] and references therein. The non-standard finite difference (NSFD) schemes were firstly proposed by Mickens [10], both for ordinary differential equations (ODEs) and partial differential equations (PDEs) with more accuracy than standard finite difference method (SFDM).

Recently, many interesting studies were presented to the stochastic tuberculosis (TB) model such as [11-14]. Also, several papers considered modeling tuberculosis (TB) [15-20]; however, the proposed model introduces several factors in spreading TB, for 
instance, the fast infection, the exogenous reinfection, and secondary infection along with the resistance factor [21].

The aim of this paper is to develop an accurate numerical algorithm for approximating the numerical solutions of the stochastic multi-strain TB model. This scheme is based on the rules introduced in the deterministic case by R.E. Mickens (see [10, 22-24, 28, 32]). A new technique is called a nonstandard theta Milstein method (NTMM). It is constructed to illustrate the behavior of the solutions of SDE with various value of $\theta$. Mean-square (MS) stability of nonstandard theta Milstein method is studied.

On the other hand, mathematical models are considered as important tools to describe several problems in natural sciences such as biology, physics, and meteorology $([15,25-32])$.

To the best of our knowledge, the NTMM for solving the stochastic multi-strain TB model has never been explored.

This paper is organized as follows. In the "A stochastic multi-strain TB model" section, a stochastic multi-strain TB model is constructed. In the "Stability of the SDE model for multi-strain tuberculosis model" section, the stability of the SDE model for multistrain TB model is proved. In the "Basic properties of the solution" section, NTMM is constructed to solve a stochastic multi-strain TB model, and the mean-square stability of NTMM is proved in the "Mean-square stability of NSTMM" section. The numerical implementation of the proposed technique is given in the "Simulations" section. In the "Conclusions" section, the conclusions are given.

\section{A stochastic multi-strain TB model}

In this section, we introduce the multi-strain TB model which is given in [33]; this model incorporates three strains: drug-sensitive, emerging multi-drug-resistant (MDR), and extensively drug-resistant (XDR). The population of interest is divided into eight compartments, see Table 1 . Let us assume that $\left(\Omega, \digamma,\left\{\digamma_{t}\right\}_{t \geq t_{0}}, \mathbb{P}\right)$ is a complete probability space with a filtration $\left\{\digamma_{t}\right\}_{t \geq t_{0}}$. Let $W(t)$ be an eight-dimensional Wiener process defined on this probability space. We assume that the eight coordinates $W_{1}(t), W_{2}(t), \ldots, W_{8}(t)$ are mutually independent and $\xi_{1}, \xi_{2}, \ldots, \xi_{8}$ represent the intensities of the white noises. The stochastic perturbation in our model is a white noise type that is directly proportional to the all variables of the model. Therefore, the stochastic system can be described by the It $\hat{o}$ system as follows:

Table 1 All variables in system (1)-(8) and their definitions

\begin{tabular}{ll}
\hline Variable & Definition \\
\hline$S(t)$ & The susceptible population (individuals who have never encountered TB) \\
$L_{S}(t)$ & The individuals infected with the drug-sensitive TB strain but who are \\
& in a latent stage, i.e., who are neither showing symptoms nor infecting others \\
$L_{m}(t)$ & Individuals latently infected with MDR-TB \\
$L_{X}(t)$ & Individuals latently infected with XDR-TB \\
$I_{S}(t)$ & Individuals infected with the drug-sensitive TB strain who are infectious \\
& to others (and most likely showing symptoms as well) \\
$I_{m}(t)$ & Individuals who are infectious with the MDR-TB strain \\
$I_{x}(t)$ & Individuals who are infectious with the XDR-TB strain \\
$R(t)$ & Individuals for whom treatment was successful \\
$N(t)$ & The total population, \\
& $N=S+L_{s}+L_{m}+L_{x}+I_{s}+I_{m}+I_{x}+R$. \\
\hline
\end{tabular}




$$
\begin{aligned}
& d S(t)=\left(b-d S-\beta_{s} \frac{S I_{s}}{N}-\beta_{m} \frac{S I_{m}}{N}-\beta_{x} \frac{S I_{x}}{N}\right) d t+\xi_{1} S d W_{1}(t), \\
& d L_{s}(t)=\left(\lambda_{s} \beta_{s} \frac{S I_{s}}{N}+\sigma_{s} \lambda_{s} \beta_{s} \frac{R I_{s}}{N}-\alpha_{s s} \beta_{s} \frac{L_{s} I_{s}}{N}-\alpha_{s m} \beta_{m} \frac{L_{s} I_{m}}{N}\right. \\
& \left.-\alpha_{s x} \beta_{x} \frac{L_{s} I_{x}}{N}+\gamma_{s} I_{s}-\left(d+\epsilon_{s}+t_{1 s}\right) L_{s}\right) d t+\xi_{2} L_{s} d W_{2}(t) \\
& d L_{m}(t)=\left(\lambda_{m} \beta_{m} \frac{S I_{m}}{N}+\sigma_{m} \lambda_{m} \beta_{m} \frac{R I_{m}}{N}+\alpha_{s m} \beta_{m} \lambda_{m} \frac{L_{s} I_{m}}{N}-\alpha_{m m} \beta_{m} \frac{L_{m} I_{m}}{N}\right. \\
& -\alpha_{m x} \beta_{x} \frac{L_{m} I_{x}}{N}+\gamma_{m} I_{m}-\left(d+\epsilon_{m}\right) L_{m}+t_{1 s} L_{s}-P_{1} t_{1 s} L_{s}+t_{2 s} I_{s} \\
& \left.-P_{2} t_{2 s} I_{s}\right) d t+\xi_{3} L_{m} d W_{3}(t) \\
& d L_{x}(t)=\left(\lambda_{x} \beta_{x} \frac{S I_{x}}{N}+\sigma_{x} \lambda_{x} \beta_{x} \frac{R I_{x}}{N}+\alpha_{s x} \beta_{x} \lambda_{x} \frac{L_{s} I_{x}}{N}+\alpha_{m x} \beta_{x} \lambda_{x} \frac{L_{m} I_{x}}{N}\right. \\
& \left.-\alpha_{x x} \beta_{x} \frac{L_{x} I_{x}}{N}-\left(d+\epsilon_{x}\right) L_{x}+\gamma_{x} I_{x}+t_{2 m} I_{m}-P_{3} t_{2 m} I_{m}\right) d t \\
& +\xi_{4} L_{x} d W_{4}(t) \\
& d I_{s}=\alpha_{s s} \beta_{s} \frac{L_{s} I_{s}}{N}+\left(1-\lambda_{s}\right) \beta_{s}\left(\frac{S I_{s}}{N}+\sigma_{s} \frac{R I_{s}}{N}\right)+\epsilon_{s} L_{s} \\
& \left.-\left(d+\delta_{s}+t_{2 s}+\gamma_{s}\right) I_{s}\right) d t+\xi_{5} I_{s} d W_{5}(t), \\
& d I_{m}=\alpha_{m m} \beta_{m} \frac{L_{m} I_{m}}{N}+\left(1-\lambda_{m}\right) \beta_{m}\left(\frac{S I_{m}}{N}+\sigma_{m} \frac{R I_{m}}{N}+\alpha_{s m} \frac{L_{s} I_{m}}{N}\right) \\
& \left.+\epsilon_{m} L_{m}-\left(d+\delta_{m}+t_{2 m}+\gamma_{m}\right) I_{m}\right) d t+\xi_{6} I_{m} d W_{6}(t), \\
& d I_{x}(t)=\left(\alpha_{x x} \beta_{x} \frac{L_{x} I_{x}}{N}+\left(1-\lambda_{x}\right) \beta_{x}\left(\frac{S I_{x}}{N}+\sigma_{x} \frac{R I_{x}}{N}+\alpha_{s x} \frac{L_{s} I_{x}}{N}+\alpha_{m x} \frac{L_{m} I_{x}}{N}\right)\right. \\
& \left.+\epsilon_{x} L_{x}-\left(d+\delta_{x}+t_{2 x}+\gamma_{x}\right) I_{x}\right) d t+\xi_{7} I_{x} d W_{7}(t), \\
& d R=P_{1} t_{1 s} L_{s}+P_{2} t_{2 s} I_{s}+P_{3} t_{2 m} I_{m}+t_{2 x} I_{x}-\sigma_{s} \beta_{s} \frac{R I_{s}}{N} \\
& \left.-\sigma_{m} \beta_{m} \frac{R I_{m}}{N}-\sigma_{x} \beta_{x} \frac{R I_{x}}{N}-d R\right) d t+\xi_{8} R d W_{8}(t) .
\end{aligned}
$$

To investigate the dynamical behavior of the proposed model, the first thing is whether we have a unique global solution (i.e., no explosion in a finite time) for any given initial value. Consider the following $d$-dimensional stochastic system:

$$
d X(t)=F(t, X(t)) d t+G(t, X(t)) d W(t)
$$

where $F(t, X(t))$ is a function in $R^{d}$ defined in $\left[t_{0}, \infty[\times R\right.$ and $G(t, X(t))$ is a $d \times m$ matrix, $F$ and $G$ are locally Lipschitz functions in $X$, and $W=W(t)_{t \geq 0}$ is a $d$-dimensional Wiener process. We assume that $X=0$ is a solution of system (1)-(8). The coefficients of Eq. (9) are generally required to satisfy linear growth conditions and local Lipschitz conditions [34-36]. If the coefficients $F$ and $G$ of (9) do not satisfy linear growth conditions, the solution of system (9) may explode at a finite time. From [36], the coefficients are locally Lipschitz. Consequently, the system has a unique local solution for any feasible initial state.

In order to study stability of disease-free equilibrium point $E_{*}$, we assume that there are global solutions which are almost surely non-negative. 


\section{Stability of the SDE model for multi-strain tuberculosis model}

In this section, the theorem below can be interpreted as follows; at least, the stochastic perturbations do not destabilize the system. Let $\left(R_{0 s}, R_{0 m}, R_{0 x}\right)$ the basic reproduction number of drug-sensitive strain, MDR strain, and XDR strain. Let us define $R_{*}$ as follows:

$$
\begin{aligned}
R_{*} & =\max \left(R_{0 s}, R_{0 m}, R_{0 x}\right), \text { where } \\
R_{0 s} & =\frac{\beta_{s}\left(\varepsilon_{s}+\left(1-\lambda_{s}\right)\left(d+t_{1 s}\right)\right)}{\left(\varepsilon_{s}+d+t_{1 s}\right)\left(t_{2 s}+\delta_{s}+d\right)+\gamma_{s}\left(t_{1 s}+d\right)}, \\
R_{0 m} & =\frac{\beta_{m}\left(\varepsilon_{m}+\left(1-\lambda_{m}\right) d\right)}{\left(\varepsilon_{m}+d\right)\left(t_{2 m}+\delta_{m}+d\right)+d \gamma_{m}}, \\
R_{0 x} & =\frac{\beta_{x}\left(\varepsilon_{x}+\left(1-\lambda_{x}\right) d\right)}{\left(\varepsilon_{x}+d\right)\left(t_{2 x}+\delta_{x}+d\right)+d \gamma_{x}} .
\end{aligned}
$$

Theorem 1 If $R_{*}<1$, then disease-free equilibrium is a.s exponentially stable.

Proof Assume that [33]:

$$
\begin{aligned}
& 0 \leq \alpha_{s s} \leq\left(1-\lambda_{s}\right), \\
& 0 \leq \alpha_{m m} \leq\left(1-\lambda_{m}\right), \\
& 0 \leq \alpha_{x x} \leq\left(1-\lambda_{x}\right) .
\end{aligned}
$$

Using the fact that $\frac{S(t)+\sigma_{S} R(t)}{N}<1$ and that $I_{s}(t) \leq I_{s}^{\infty}$ at any $t$, it follows

$$
L_{s}^{\infty} \leq \frac{\lambda_{s} \beta_{s}+\gamma_{s}}{d+t_{1 s}+\epsilon_{s}} I_{s}^{\infty}
$$

Using assumption (11), and for simplicity, let us define $a_{1}:=\left(d+\delta_{s}+t_{2 s}+\gamma_{s}\right), a_{2}:=$ $\left.\left(d+\epsilon_{s}+t_{1 s}\right)\right)$. The fact that $\frac{S(t)+\sigma_{s} R(t)+L_{s}(t)}{N}<1$ and that $L_{s}(t) \leq L_{s}^{\infty}$, together with Eq. (14), implies that

$$
0 \leq\left[R_{0 s}-1\right] \frac{1}{a_{2}\left(a_{2} a_{1}-\epsilon_{s} \gamma_{s}\right)} I_{s}^{\infty},
$$

since $R_{*}=\max \left(R_{0 s}, R_{0 m}, R_{0 x}\right), \quad R_{*}<1$ implies that $R_{0 s}<1$. Similarly, using assumptions (12) and (13), we can prove the following inequalities involving $I_{m}$ and $I_{x}$. Define a $\mathbb{C}^{8}$ function $V: \mathbb{R}_{+}^{8} \rightarrow \mathbb{R}_{+}$by $V\left(S(t), L_{s}(t), L_{m}(t), L_{x}(t), I_{s}(t), I_{m}(t), I_{x}(t), R(t)\right)$ which is a non-negativity function. $V(t)=\left(S(t)+L_{s}(t)+L_{m}(t)+L_{x}(t)+I_{s}(t)+I_{m}(t)+I_{x}(t)+R(t)\right)$. Thus, we can define $Z=\ln V$ by the Itô formula; we compute,

$$
\begin{aligned}
L V & =\frac{1}{V} d S+\frac{1}{V} d L_{s}+\frac{1}{V} d L_{m}+\frac{1}{V} d L_{x}+\frac{1}{V} d I_{s}+\frac{1}{V} d I_{m}+\frac{1}{V} d I_{x}+\frac{1}{V} d R \\
& -\frac{1}{2}\left[\frac{1}{V^{2}} d S^{2}+\frac{1}{V^{2}} d L_{s}^{2}+\frac{1}{V^{2}} d L_{m}^{2}+\frac{1}{V^{2}} d L_{x}^{2}+\frac{1}{V^{2}} d I_{s}^{2}+\frac{1}{V^{2}} d I_{m}^{2}+\frac{1}{V^{2}} d I_{x}^{2}+\frac{1}{V^{2}} d R^{2}\right] \\
& =\left(b-d S-\beta_{s} \frac{S I_{s}}{N}-\beta_{m} \frac{S I_{m}}{N}-\beta_{x} \frac{S I_{x}}{N}\right) V^{-1} d t
\end{aligned}
$$




$$
\begin{aligned}
& +\left(\lambda_{s} \beta_{s} \frac{S I_{s}}{N}+\sigma_{s} \lambda_{s} \beta_{s} \frac{R I_{s}}{N}-\alpha_{s s} \beta_{s} \frac{L_{s} I_{s}}{N}-\alpha_{s m} \beta_{m} \frac{L_{s} I_{m}}{N}-\alpha_{s x} \beta_{x} \frac{L_{s} I_{x}}{N}+\gamma_{s} I_{s}\right) \\
& \left.-\left(d+\epsilon_{s}+t_{1 s}\right) L_{s}\right) V^{-1} d t \\
& +\left(\lambda_{m} \beta_{m} \frac{S I_{m}}{N}+\sigma_{m} \lambda_{m} \beta_{m} \frac{R I_{m}}{N}+\alpha_{s m} \beta_{m} \lambda_{m} \frac{L_{s} I_{m}}{N}-\alpha_{m m} \beta_{m} \frac{L_{m} I_{m}}{N}-\alpha_{m x} \beta_{x} \frac{L_{m} I_{x}}{N}\right) \\
& \left.+\gamma_{m} I_{m}-\left(d+\varepsilon_{m}\right) L_{m}+\left(1-P_{1}\right) t_{1 s} L_{s}+\left(1-P_{2}\right) t_{2 s} I_{s}\right) V^{-1} d t \\
& +\left(\lambda_{x} \beta_{x} \frac{S I_{x}}{N}+\sigma_{x} \lambda_{x} \beta_{x} \frac{R I_{x}}{N}+\alpha_{s x} \beta_{x} \lambda_{x} \frac{L_{s} I_{x}}{N}+\alpha_{m x} \beta_{x} \lambda_{x} \frac{L_{m} I_{x}}{N}\right. \\
& \left.-\alpha_{x x} \beta_{x} \frac{L_{x} I_{x}}{N}-\left(d+\varepsilon_{x}\right) L_{x}+\gamma_{x} I_{x}+\left(1-P_{3}\right) t_{2 m} I_{m}\right) V^{-1} d t \\
& +\left(\alpha_{s s} \beta_{s} \frac{L_{s} I_{s}}{N}+\left(1-\lambda_{s}\right) \beta_{s}\left(\frac{S I_{s}}{N}+\sigma_{s} \frac{R I_{s}}{N}\right)+\varepsilon_{s} L_{s}\right. \\
& \left.-\left(d+\delta_{s}+t_{2 s}+\gamma_{s}\right) I_{s}\right) V^{-1} d t \\
& +\left(1-\lambda_{m}\right) \beta_{m}\left(\frac{S I_{m}}{N}+\sigma_{m} \frac{R I_{m}}{N}+\alpha_{s m} \frac{L_{s} I_{m}}{N}\right)+\alpha_{m m} \beta_{m} \frac{L_{m} I_{m}}{N}+\varepsilon_{m} L_{m} \\
& \left.-\left(d+\delta_{m}+t_{2 m}+\gamma_{m}\right) I_{m}\right) V^{-1} d t \\
& +\left(\alpha_{x x} \beta_{x} \frac{L_{x} I_{x}}{N}+\left(1-\lambda_{x}\right) \beta_{x}\left(\frac{S I_{x}}{N}+\sigma_{x} \frac{R I_{x}}{N}+\alpha_{s x} \frac{L_{s} I_{x}}{N}+\alpha_{m x} \frac{L_{m} I_{x}}{N}\right)+\epsilon_{x} L_{x}\right. \\
& \left.-\left(d+\delta_{x}+t_{2 x}+\gamma_{x}+\epsilon_{4} u_{4}(t)\right) I_{x}\right) V^{-1} d t \\
& +\left(P_{1} t_{1 s} L_{s}+P_{2} t_{2 s} I_{s}+P_{3} t_{2 m} I_{m}+t_{2 x} I_{x}+\epsilon_{4} u_{4}(t) I_{x}-\sigma_{s} \beta_{s} \frac{R I_{s}}{N}\right. \\
& \left.-\sigma_{m} \beta_{m} \frac{R I_{m}}{N}-\sigma_{x} \beta_{x} \frac{R I_{x}}{N}-d R\right) V^{-1} d t-\frac{1}{2}\left[\frac{\xi_{1}^{2} S}{V^{2}}+\frac{\xi_{2}^{2} L_{s}}{V^{2}}+\frac{\xi_{3}^{2} L_{m}}{V^{2}}+\frac{\xi_{4}^{2} L_{x}}{V^{2}}\right. \\
& \left.+\frac{\xi_{5}^{2} I_{s}}{V^{2}}+\frac{\xi_{6}^{2} I_{m}}{V^{2}}+\frac{\xi_{7}^{2} I_{x}}{V^{2}}+\frac{\xi_{8}^{2} R}{V^{2}}\right] .
\end{aligned}
$$

Then,

$$
\begin{aligned}
d Z & =L V d t+\left[\xi_{1} S d W_{1}+\xi_{2} L_{s} d W_{2}+\xi_{3} L_{m} d W_{3}+\xi_{4} L_{x} d W_{4}+\xi_{5} I_{s} d W_{5}\right. \\
& \left.+\xi_{6} I_{m} d W_{6}+\xi_{7} I_{x} d W_{7}+\xi_{8} R d W_{8}\right] V^{-1} .
\end{aligned}
$$

Therefore,

$$
Z=Z_{0}+\int_{0}^{t} L V d t+\sum_{i=1}^{8} G_{i}(t)
$$

where each $G_{i}(t)$ is a martingale defined as: $G_{1}(t)=\int_{0}^{t} \frac{\xi_{1} S d W_{1}}{V}, G_{2}(t)=\int_{0}^{t} \frac{\xi_{2} L_{s} d W_{2}}{V}$,

$$
\begin{aligned}
& G_{3}(t)=\int_{0}^{t} \frac{\xi_{3} L_{m} d W_{2}}{V}, G_{4}(t)=\int_{0}^{t} \frac{\xi_{4} L_{x} d W_{4}}{V}, G_{5}(t)=\int_{0}^{t} \frac{\xi_{5} I_{s} d W_{5}}{V}, G_{6}(t)=\int_{0}^{t} \frac{\xi_{6} I_{m} d W_{6}}{V}, \\
& G_{7}(t)=\int_{0}^{t} \frac{\xi_{7} I_{x} d W_{7}}{V}, G_{8}(t)=\int_{0}^{t} \frac{\xi_{8} R d W_{8}}{V} \text {. Then, } \\
& \lim _{t \rightarrow \infty} \frac{Z}{t}=\lim _{t \rightarrow \infty} \frac{Z_{0}}{t}+\lim _{t \rightarrow \infty} \frac{1}{t} \int_{0}^{t} L V d t \\
& +\lim _{t \rightarrow \infty} \frac{1}{t} \sum_{i=1}^{8} G_{i}(t) .
\end{aligned}
$$


Regarding the quadratic variations of the stochastic integral $G_{i}(t)$, we have $\int_{0}^{t} \frac{\left(\xi_{1} S\right)^{2}}{V^{2}} d s \leq$ $\xi_{1}^{2} t, \quad \int_{0}^{t} \frac{\left(\xi_{2} L_{s}\right)^{2}}{V^{2}} d s \leq \xi_{2}^{2} t, \quad \int_{0}^{t} \frac{\left(\xi_{3} L_{m}\right)^{2}}{V^{2}} d s \leq \xi_{3}^{2} t, \quad \int_{0}^{t} \frac{\left(\xi_{4} L_{x}\right)^{2}}{V^{2}} d s \leq \xi_{4}^{2} t, \quad \int_{0}^{t} \frac{\left(\xi_{5} I_{s}\right)^{2}}{V^{2}} d s \leq \xi_{5}^{2} t$, $\int_{0}^{t} \frac{\left(\xi_{6} I_{m}\right)^{2}}{V^{2}} d s \leq \xi_{6}^{2} t, \quad \int_{0}^{t} \frac{\left(\xi_{7} I_{x}\right)^{2}}{V^{2}} d s \leq \xi_{7}^{2} t, \quad \int_{0}^{t} \frac{\left(\xi_{8} R\right)^{2}}{V^{2}} d s \leq \xi_{8}^{2} t$. By the strong law of large numbers for martingales [36], we therefore have

$$
\lim _{t \rightarrow \infty} \sup \frac{1}{t} \sum_{i=1}^{8} G_{i}(t)=0 \quad \text { (a.s). }
$$

It finally follows from (16) by dividing $t$ on both sides and then letting $t \rightarrow \infty$ that

$$
\lim _{t \rightarrow \infty} \sup \frac{\ln Z(t)}{t} \leq \lim _{t \rightarrow \infty} \sup \frac{1}{t} \int_{0}^{t} L V d t \quad(\text { a.s }) .
$$

We note that

$$
\begin{aligned}
& L V \leq \frac{1}{V} d S+\frac{1}{V} d L_{s}+\frac{1}{V} d L_{m}+\frac{1}{V} d L_{x}+\frac{1}{V} d I_{s}+\frac{1}{V} d I_{m}+\frac{1}{V} d I_{x}+\frac{1}{V} d R \\
& -\frac{1}{2}\left[\frac{1}{V^{2}} d S^{2}+\frac{1}{V^{2}} d L_{s}^{2}+\frac{1}{V^{2}} d L_{m}^{2}+\frac{1}{V^{2}} d L_{x}^{2}+\frac{1}{V^{2}} d I_{s}^{2}+\frac{1}{V^{2}} d I_{m}^{2}+\frac{1}{V^{2}} d I_{x}^{2}+\frac{1}{V^{2}} d R^{2}\right] \\
& =\left(b-d S-\beta_{s} \frac{S I_{s}}{N}-\beta_{m} \frac{S I_{m}}{N}-\beta_{x} \frac{S I_{x}}{N}\right) V^{-1} d t \\
& +\left(\lambda_{s} \beta_{s} \frac{S I_{s}}{N}+\sigma_{s} \lambda_{s} \beta_{s} \frac{R I_{s}}{N}-\alpha_{s s} \beta_{s} \frac{L_{s} I_{s}}{N}-\alpha_{s m} \beta_{m} \frac{L_{s} I_{m}}{N}-\alpha_{s x} \beta_{x} \frac{L_{s} I_{x}}{N}+\gamma_{s} I_{s}\right) \\
& \left.-\left(d+\epsilon_{s}+t_{1 s}\right) L_{s}\right) V^{-1} d t \\
& +\left(\lambda_{m} \beta_{m} \frac{S I_{m}}{N}+\sigma_{m} \lambda_{m} \beta_{m} \frac{R I_{m}}{N}+\alpha_{s m} \beta_{m} \lambda_{m} \frac{L_{s} I_{m}}{N}-\alpha_{m m} \beta_{m} \frac{L_{m} I_{m}}{N}-\alpha_{m x} \beta_{x} \frac{L_{m} I_{x}}{N}\right) \\
& \left.+\gamma_{m} I_{m}-\left(d+\varepsilon_{m}\right) L_{m}+\left(1-P_{1}\right) t_{1 s} L_{s}+\left(1-P_{2}\right) t_{2 s} I_{s}\right) V^{-1} d t \\
& +\left(\lambda_{x} \beta_{x} \frac{S I_{x}}{N}+\sigma_{x} \lambda_{x} \beta_{x} \frac{R I_{x}}{N}+\alpha_{s x} \beta_{x} \lambda_{x} \frac{L_{s} I_{x}}{N}+\alpha_{m x} \beta_{x} \lambda_{x} \frac{L_{m} I_{x}}{N}\right. \\
& \left.-\alpha_{x x} \beta_{x} \frac{L_{x} I_{x}}{N}-\left(d+\varepsilon_{x}\right) L_{x}+\gamma_{x} I_{x}+\left(1-P_{3}\right) t_{2 m} I_{m}\right) V^{-1} d t \\
& +\left(\alpha_{s s} \beta_{s} \frac{L_{s} I_{s}}{N}+\left(1-\lambda_{s}\right) \beta_{s}\left(\frac{S I_{s}}{N}+\sigma_{s} \frac{R I_{s}}{N}\right)+\varepsilon_{s} L_{s}-\left(d+\delta_{s}+t_{2 s}+\gamma_{s}\right) I_{s}\right) V^{-1} d t \\
& +\left(\left(1-\lambda_{m}\right) \beta_{m}\left(\frac{S I_{m}}{N}+\sigma_{m} \frac{R I_{m}}{N}+\alpha_{s m} \frac{L_{s} I_{m}}{N}\right)+\alpha_{m m} \beta_{m} \frac{L_{m} I_{m}}{N}+\varepsilon_{m} L_{m}\right. \\
& \left.-\left(d+\delta_{m}+t_{2 m}+\gamma_{m}\right) I_{m}\right) V^{-1} d t \\
& +\left(\alpha_{x x} \beta_{x} \frac{L_{x} I_{x}}{N}+\left(1-\lambda_{x}\right) \beta_{x}\left(\frac{S I_{x}}{N}+\sigma_{x} \frac{R I_{x}}{N}+\alpha_{s x} \frac{L_{s} I_{x}}{N}+\alpha_{m x} \frac{L_{m} I_{x}}{N}\right)+\epsilon_{x} L_{x}\right. \\
& \left.-\left(d+\delta_{x}+t_{2 x}+\gamma_{x}+\epsilon_{4} u_{4}(t)\right) I_{x}\right) V^{-1} d t \\
& +\left(P_{1} t_{1 s} L_{s}+P_{2} t_{2 s} I_{s}+P_{3} t_{2 m} I_{m}+t_{2 x} I_{x}+\epsilon_{4} u_{4}(t) I_{x}-\sigma_{s} \beta_{s} \frac{R I_{s}}{N}\right. \\
& \left.-\sigma_{m} \beta_{m} \frac{R I_{m}}{N}-\sigma_{x} \beta_{x} \frac{R I_{x}}{N}-d R\right) V^{-1} d t-\frac{1}{2}\left[\frac{\xi_{1}^{2} S}{V^{2}}+\frac{\xi_{2}^{2} L_{s}}{V^{2}}+\frac{\xi_{3}^{2} L_{m}}{V^{2}}+\frac{\xi_{4}^{2} L_{x}}{V^{2}}\right. \\
& \left.+\frac{\xi_{5}^{2} I_{s}}{V^{2}}+\frac{\xi_{6}^{2} I_{m}}{V^{2}}+\frac{\xi_{7}^{2} I_{x}}{V^{2}}+\frac{\xi_{8}^{2} R}{V^{2}}\right]
\end{aligned}
$$


and in fact

$$
\lim _{t \rightarrow \infty} \sup \frac{1}{t} \int_{0}^{t} L V d t<0,
$$

therefore,

$$
\lim _{t \rightarrow \infty} \sup \frac{\ln Z(t)}{t}<0 .
$$

This finally proves the (a.s.) exponential stability.

\section{Basic properties of the solution}

In this section, we remind classical results about continuous and discrete stochastic differential equation systems.

\section{Stochastic boundedness}

Stochastic boundedness is one of the most important topics because boundedness of a system guarantees its validity in a population system. We first present the definition of stochastically ultimate boundedness.

Definition 1 (see [37]) The solution

$$
X(t)=\left(S(t), L_{s}(t), L_{m}(t), L_{x}(t), I_{s}(t), I_{m}(t), I_{x}(t), R(t)\right),
$$

of Eqs. (1)-(8) is said to be stochastically ultimately bounded if for any $\epsilon \in(0,1)$, there is a positive constant $\delta=\delta(\epsilon)$ such that for any initial value $X_{0} \in R_{+}^{8}$, the solution $X(t)$ to (1)-(8) has the property that:

$$
\lim _{t \rightarrow \infty} \sup P\{|X(t)|>\delta\}<\epsilon .
$$

\section{Numerical method for solving stochastic model}

\section{A nonstandard theta Milstein method}

One of the merits of NFDM which is given by Mickens in 1980 [10, 22-24, 28, 32] is used to study numerically the behavior of the ordinary differential equations (ODE) and PDE efficiently. The NFDM is able to maintain the properties of the exact solution of the original ODE or PDE. The numerical scheme is called NFDM if at least one of the following conditions are satisfied [10-15, 22-28]:

1. The nonlocal approximation is used.

2. The discretization of the derivative is not traditional and uses a nonnegative function.

In this paper, we consider numerical methods for the strong solution of Itô SDEs:

$$
d z(t)=f(t, z(t)) d t+g(t, z(t)) d W(t), \quad z\left(t_{0}\right)=z_{0},
$$

where $z(t)$ is a random variable with value in $R^{m}, f: R^{m} \longrightarrow R^{m}$ is called the drift function, $g: R^{m} \longrightarrow R^{m}$ is called the diffusion function, and $d W(t)$ is a one-dimensional Wiener process, whose increment $\Delta W(t)=W(t+\Delta t)-W(t)$ is a Gaussian random variable $N(0, \triangle t)$. 
Definition 2 A general one-step theta Milstein method (TMM) is given as follows:

$$
\begin{aligned}
z_{n+1} & =z_{n}+\theta h f\left(t_{n}, z_{n}\right)+(1-\theta) h f\left(t_{n+1}, z_{n+1}\right) \\
& +g\left(t_{n}, z_{n}\right) \Delta W_{n}+\frac{1}{2} g\left(t_{n}, z_{n}\right) \frac{\partial g}{\partial z}\left(t_{n}, z_{n}\right)\left[(\triangle W)^{2}-h\right] .
\end{aligned}
$$

Definition 3 A general one-step nonstandard theta Milstein method (NTMM) is given as follows:

$$
\begin{aligned}
z_{n+1} & =z_{n}+\theta \psi(h) f\left(t_{n}, z_{n}\right)+(1-\theta) \psi(h) f\left(t_{n+1}, z_{n+1}\right) \\
& +g\left(t_{n}, z_{n}\right) \triangle W_{n}+\frac{1}{2} g\left(t_{n}, z_{n}\right) \frac{\partial g}{\partial z}\left(t_{n}, z_{n}\right)\left[(\triangle W)^{2}-h\right] .
\end{aligned}
$$

where $\varphi(h)=h+O\left(h^{2}\right)$ is a non-negative function and $\triangle W_{n}=W\left(t_{n+1}\right)-W\left(t_{n}\right)$.

\section{Special cases from NTMM:}

- If $\theta=1$, we obtain nonstandard explicit Milstein method (NEMM).

- If $\theta=0$, we obtain nonstandard drift implicit balanced Milstein method (NDIMM).

Remark 1 In (19) if $\varphi(h)=h$, then the scheme will be called theta Milstein method and its two special cases for $\theta$ are as follows:

- If $\theta=1$, we obtain explicit Milstein method (EMM).

- If $\theta=0$, we obtain the drift implicit balanced Milstein method (DIMM).

\section{Mean-square stability of NSTMM}

The mean-square stability is a stochastic version of absolute stability, and it is a very important concept in the numerical simulation of SDEs. A suitable way to find numerical schemes for SDEs is analysis of MS stability.

Definition 4 [38] The equilibrium position, $z(t) \equiv 0$, is said to be mean-square stable if for every $\varepsilon>0$ there exists $\delta_{1}>0$ such that

$$
\|z(t)\|<\varepsilon, \forall t \geq 0,\left|z_{0}\right|<\delta_{1},
$$

where $\|z(t)\|=\left(E|z(t)|^{2}\right)^{\frac{1}{2}}$.

If, in addition to (20), there exists a $\delta_{2}>0$ such that

$$
\lim _{t \rightarrow \infty}\|z(t)\|=0, \forall\left|z_{0}\right|<\delta_{2},
$$

then the equilibrium position is said to be asymptotically mean-square stable.

Definition 5 [38] Suppose that the equilibrium position of Itô's SDE as (17). The Gaussian random variable $N(0, \Delta t)$ is asymptotically mean-square stable. Then, a numerical scheme that produces the iterations $z_{n}$ to approximate the solution $z(t)$ of $(21)$ is said to be asymptotically mean-square stable if

$$
\lim _{n \rightarrow \infty}\left\|z_{n}\right\|=0 \text {. }
$$

We apply one-step scheme to the scalar linear test equation

$$
d z(t)=a z(t) d t+b z(t) d W(t), z\left(t_{0}\right)=z_{0},
$$


Table 2 All parameters and the references of system (1)-(8)

\begin{tabular}{lll}
\hline Parameter & Value & Reference \\
\hline$N$ & $20,000,30,000$ & Assumed \\
$b$ & $d N$ & {$[21]$} \\
$d$ & $1 / 73.45$ & (http://www.indexmundi.com/EGYPT/life_expectancy_at_birth.html) \\
$\beta_{s}=\beta_{m}=\beta_{x}$ & 14 & {$[40]$} \\
$\lambda_{s}=\lambda_{m}=\lambda_{x}$ & 0.5 & {$[21]$} \\
$\epsilon_{s}=\epsilon_{m}=\epsilon_{x}$ & 0.0002 & {$[21]$} \\
$\alpha_{r 1, r 2}$ & 0.05 & {$[21]$} \\
$\gamma_{s}=\gamma_{m}=\gamma_{x}$ & 0.00002 & {$[21]$} \\
$t_{1 s}$ & 2 & {$[21]$} \\
$t_{2 r}: r \in(s, m, x)$ & $t_{2 s}=2 ; t_{2 m}=t_{2 x}=1$ & {$[21]$} \\
$\sigma_{r}$ & 0.25 & {$[21]$} \\
$P_{r}$ & 0.88 & {$[21]$} \\
$\delta_{r}$ & 0 & {$[21]$} \\
$T$ & 4 years & Assumed \\
\hline
\end{tabular}

with known solution $z(t)=z_{0} e^{\left(\frac{a-b^{2}}{2}\right)}$, which is represented by

$$
z_{n+1}=R(a, b, h, J) z_{n},
$$

where $J$ is the standard Gaussian random variable $J=\frac{\Delta W_{n}}{\sqrt{h}} \sim N(0,1)$. Saito and Mitsui [39] introduced the following definition of mean-square (MS) stability.

Definition 6 [39] The numerical method is said to be MS stable for $a, b, h$ if

$$
\bar{R}(a, b, h)=E\left(R^{2}(a, b, h, J)\right)<1 .
$$

where $\bar{R}(a, b, h)$ is called MS stability function of the numerical method.

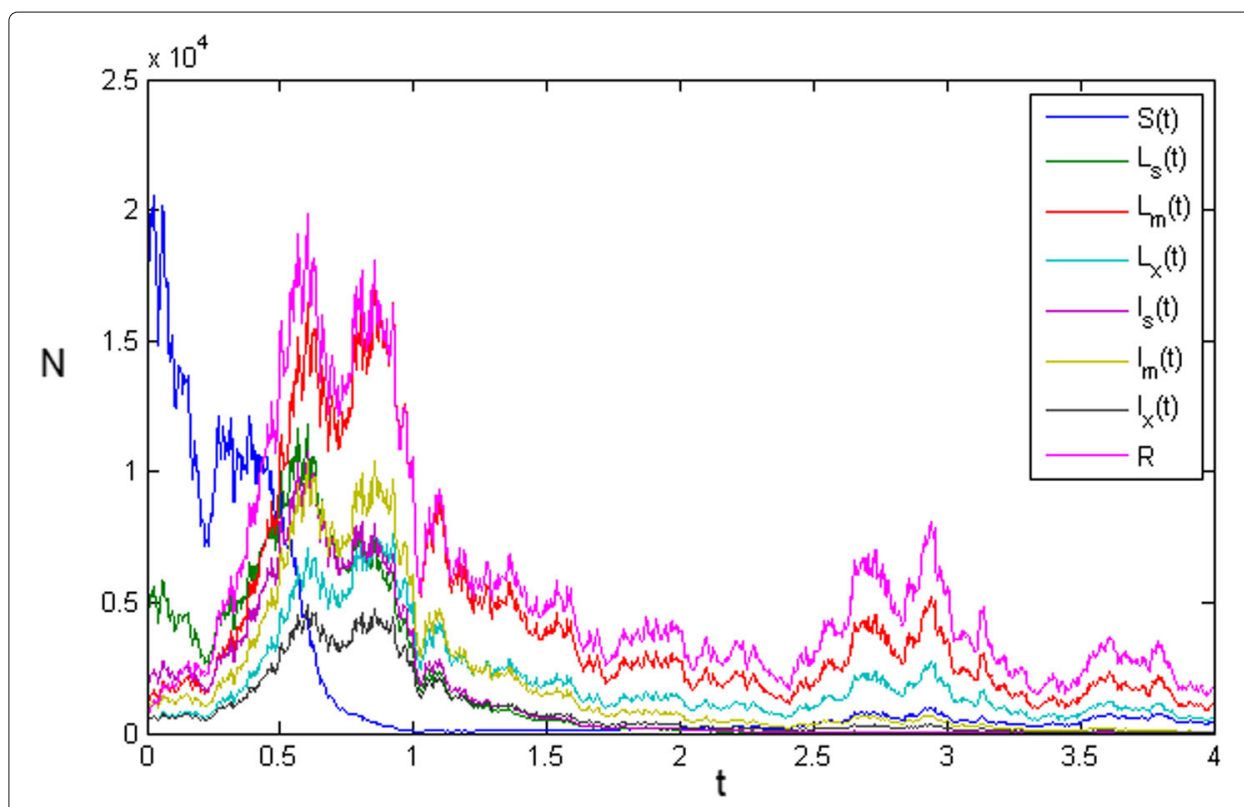

Fig. 1 Simulations of multi-strain TB model showing the various classes with $\theta=0, \xi=1$ 


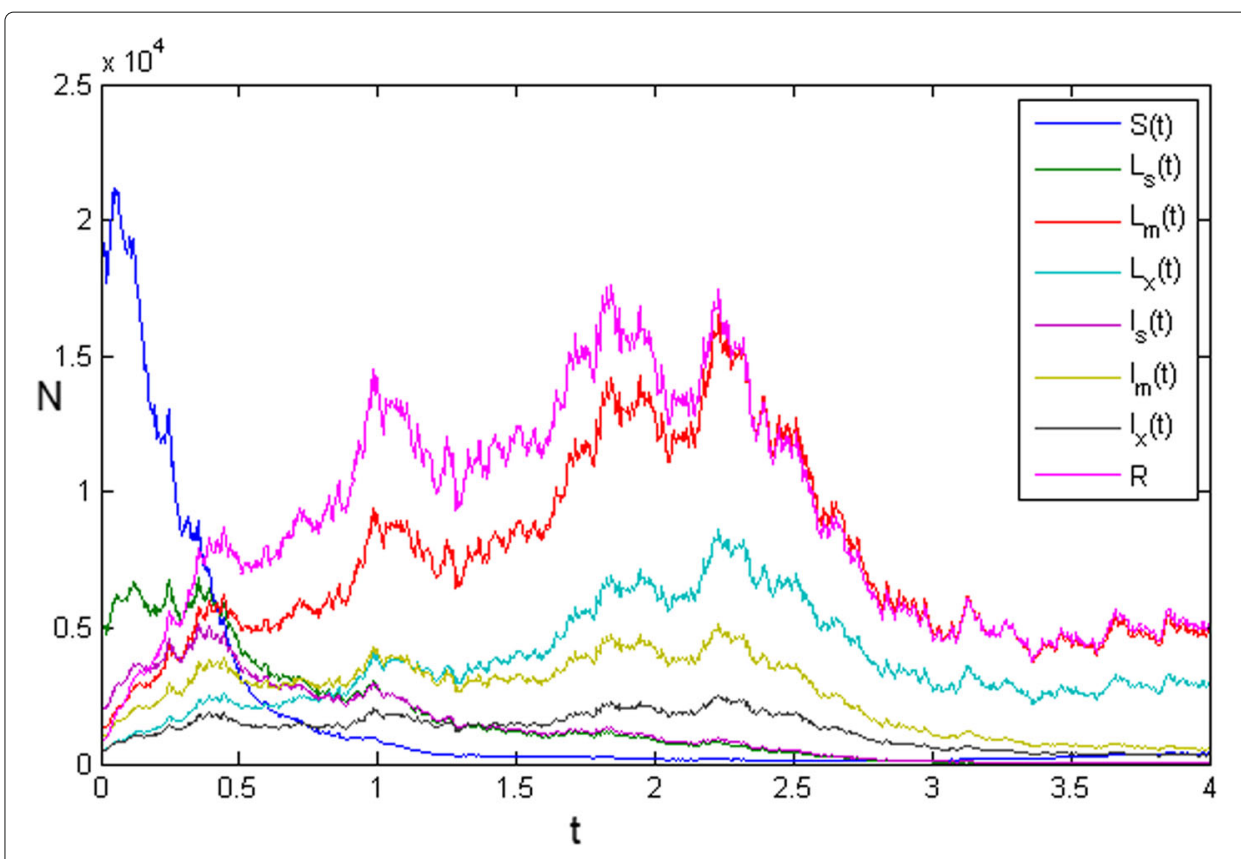

Fig. 2 Simulations of multi-strain TB model showing the various classes with $\theta=0, \xi=0.5$

For $\theta=1$ of (18), EMM is given as follows,

$$
z_{n+1}=z_{n}+f\left(t_{n}, z_{n}\right) h+g\left(t_{n}, z_{n}\right) \Delta W_{n}+0.5 g\left(t_{n}, z_{n}\right) \frac{\partial g}{\partial z}\left(t_{n}, z_{n}\right)\left[\left(\Delta W_{n}\right)^{2}-h\right] .
$$

for $\theta=0$ of (18) by introducing implicitness in $f\left(t_{n}, z_{n}\right) h$, we arrive at IDMM.

$$
\begin{aligned}
z_{n+1}= & z_{n}+f\left(t_{n+1}, z_{n+1}\right) h+g\left(t_{n}, z_{n}\right) \Delta W_{n} \\
& +0.5 g\left(t_{n}, z_{n}\right) \frac{\partial g}{\partial z}\left(t_{n}, z_{n}\right)\left[\left(\Delta W_{n}\right)^{2}-h\right] .
\end{aligned}
$$

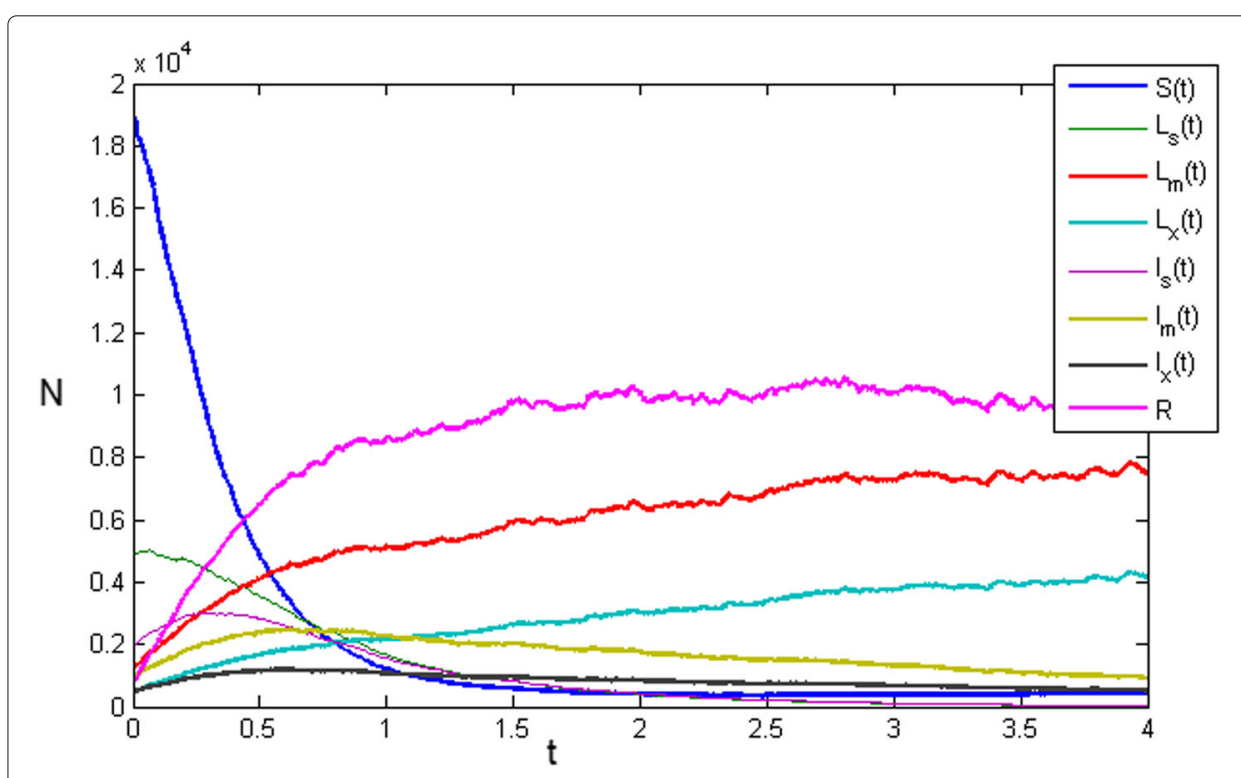

Fig. 3 Simulations of multi-strain TB model showing the various classes with $\theta=0, \xi=0.08$ 


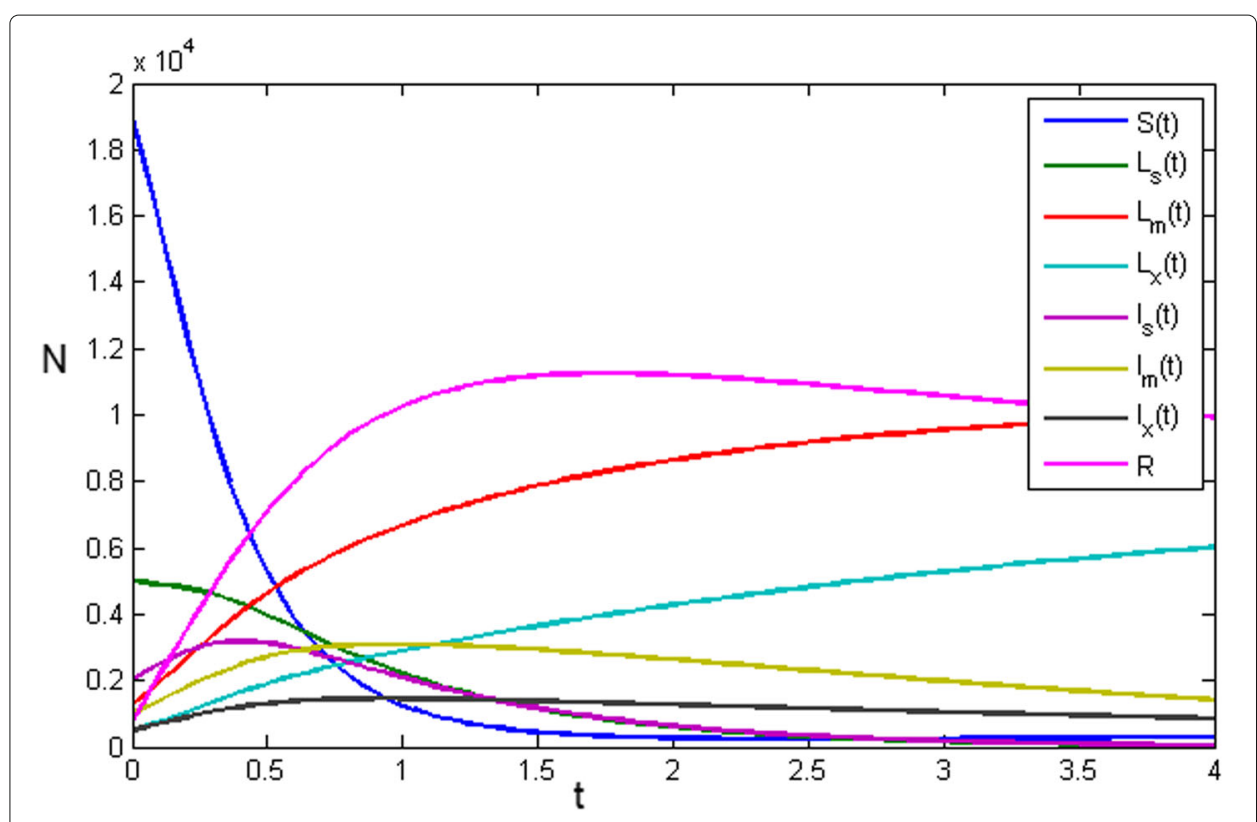

Fig. 4 Simulations of multi-strain TB model showing the various classes with $\theta=0, \xi=0$

If in (23) we replace $h$ by $\psi(h)$, we have a new method called NSIDMM; this special case from (19) when thet $a=0$ and the function $\psi(h)$ satisfies the following conditions:

$$
\varphi(h)=h+O\left(h^{2}\right), \quad 0<\varphi(h) \leq 1, \quad h \longrightarrow 0 .
$$

For more details about nonstandard method, see [10, 22-24, 28, 32] and the references cited therein. Applying the NSIDMM which given as:

$$
\begin{aligned}
z_{n+1} & =z_{n}+f\left(t_{n+1}, z_{n+1}\right) \psi(h)+g\left(t_{n}, z_{n}\right) \Delta W_{n} \\
& +0.5 g\left(t_{n}, z_{n}\right) \frac{\partial g}{\partial z}\left(t_{n}, z_{n}\right)\left[\left(\Delta W_{n}\right)^{2}-\psi(h)\right] .
\end{aligned}
$$
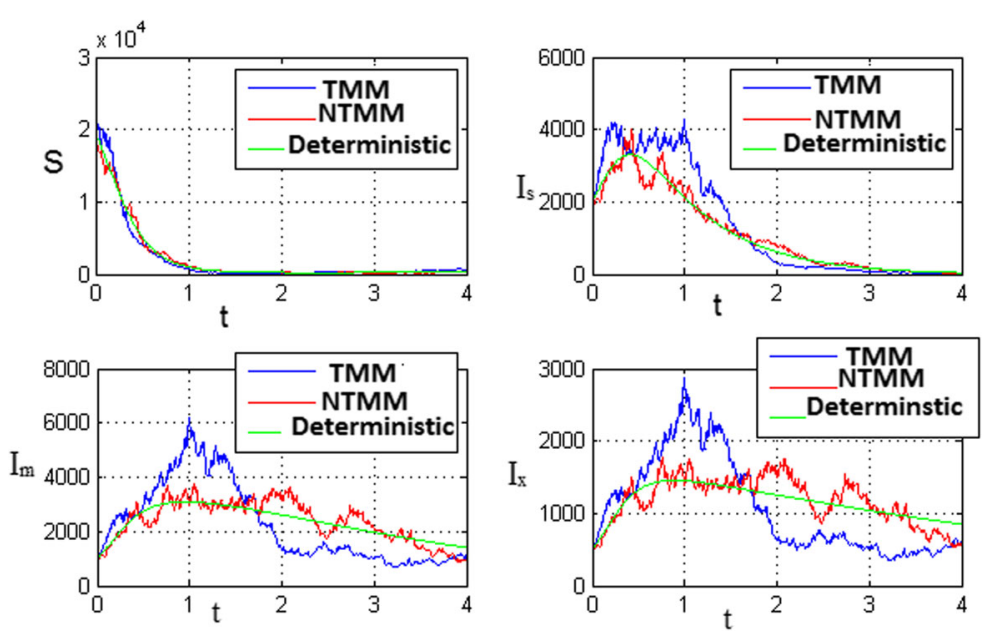

Fig. 5 The approximate solutions with $\xi=0.5, \theta=1, h=0.0067$ 

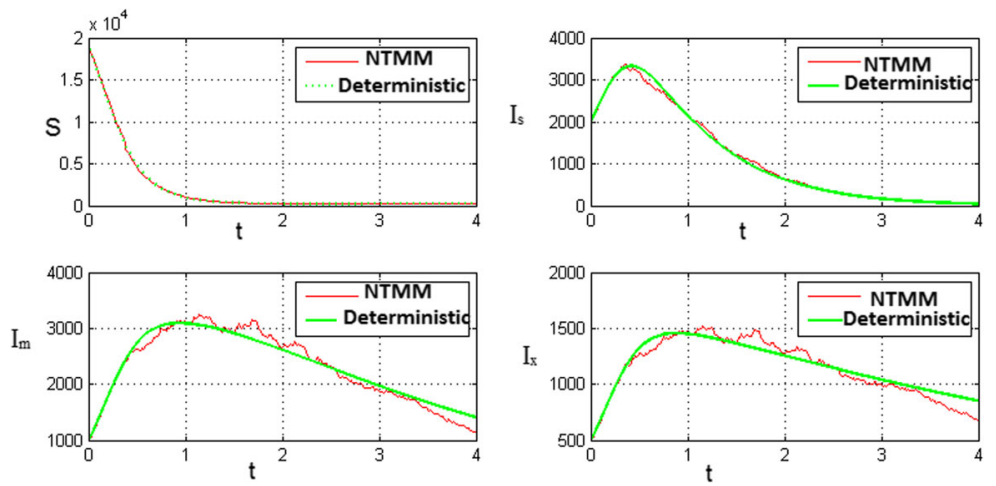

Fig. 6 Trajectories of the mean solutions of stochastic and deterministic systems with when $\theta=1, \xi=0.09$, $h=0.0133$ by using NTMM

to the linear test Eq. (21), we obtain

$$
z_{n+1}=R_{1}(p, q, J) z_{n}
$$

where $p=a \psi(h), q=b \sqrt{\psi(h)}$, and

$$
R_{1}(p, q, J)=\frac{1+q J+0.5 q^{2} J^{2}-0.5 q^{2}}{1-p} .
$$

The MS stability function of the NSDIM method is given by

$$
R_{1}(p, q)=\frac{1+q^{2}+0.5 q^{4}}{1-p} \text {. }
$$

The NSIDM will be MS stable if $R_{1}(p, q)<1$. The MS stability property of the NSIDMM is better than that of IDMM.

\section{Simulations}

In this section, we simulate the SDE model in (1)-(8) numerically for the same parameters in Table (2) and different values of $\xi$ and $\theta$. In all simulations, we use a single value for all the $\xi_{i}$, i.e., $\xi_{1}=\xi_{2}=\ldots=\xi_{8}=\xi$ with initial conditions $\left(S(0), L_{s}(0), L_{m}(0)\right.$, $\left.L_{x}(0), I_{s}(0), I_{m}(0), I_{x}(0), R(0)\right)=\left(\frac{76}{120} N, \frac{20}{120} N, \frac{5}{120} N, \frac{2}{120} N, \frac{8}{120} N, \frac{4}{120} N, \frac{2}{120} N, \frac{3}{120} N\right)$ and $\varphi(h)=Q\left(1-e^{-h}\right)$ where $Q$ is less than 0.001 . As we know, the population dynamics
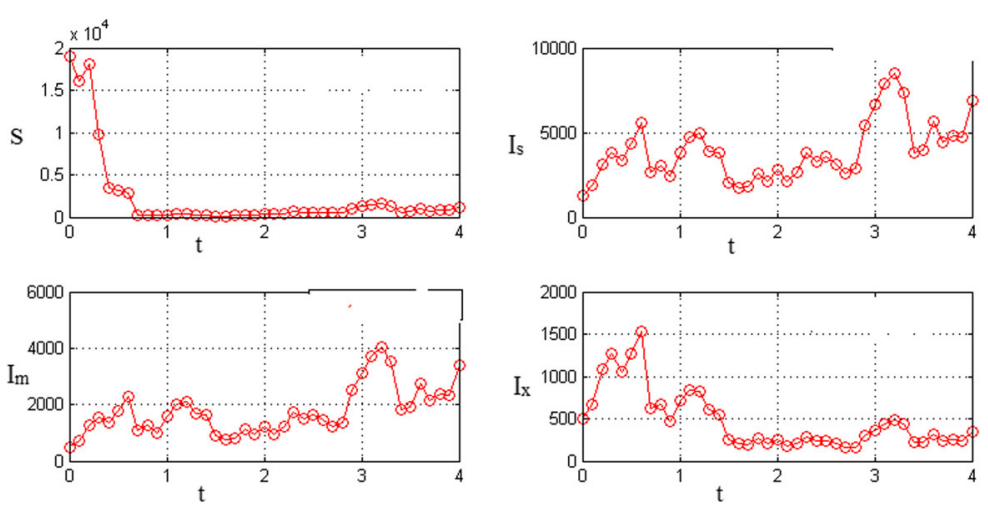

Fig. 7 The approximate solutions with $\theta=0.9, \xi=1, h=0.1$, by using NTMM 


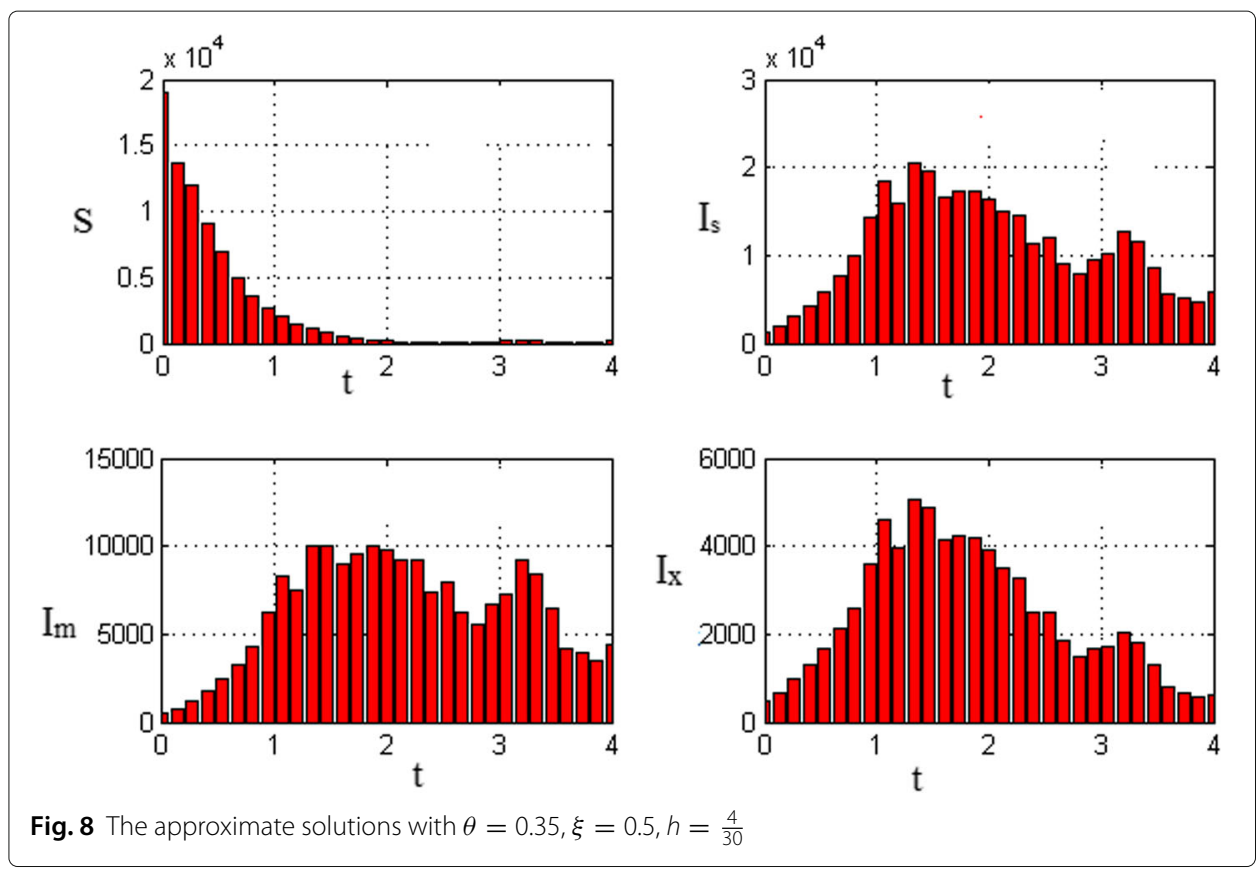

is inevitably subjected to environmental noise. So, it is important to examine the inclusion of stochastic effects into deterministic models. The simulations were run for 100,000 iterations. We use NTMM to approximate Eqs. (1)-(8); then, we have $(8 m+8)$ of nonlinear algebraic equations which can be solved using Newton's iteration method. Figures 1 , 2,3 , and 4 show the variation of $\left(S(t), L_{s}(t), L_{m}(t), L_{x}(t), I_{s}(t), I_{m}(t), I_{x}(t), R(t)\right)$ with time at different value of $\xi$ and $\theta=0$; by using the suggested technique, we note that the noise level increased when the value of $\xi$ is big. Also, in the absence of noise, we simulate the global stability of the endemic equilibrium of deterministic system (1)-(8)

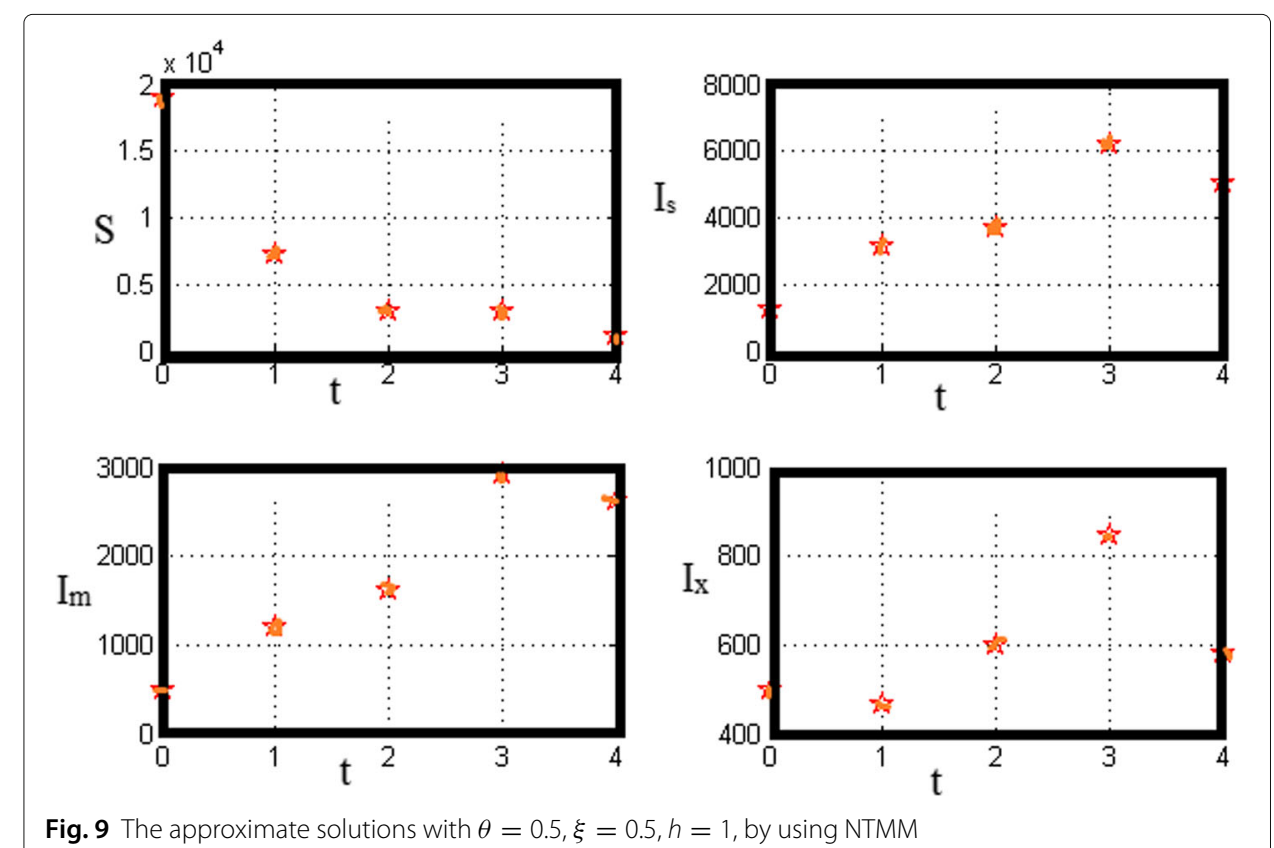

Fig. 9 The approximate solutions with $\theta=0.5, \xi=0.5, h=1$, by using NTMM 

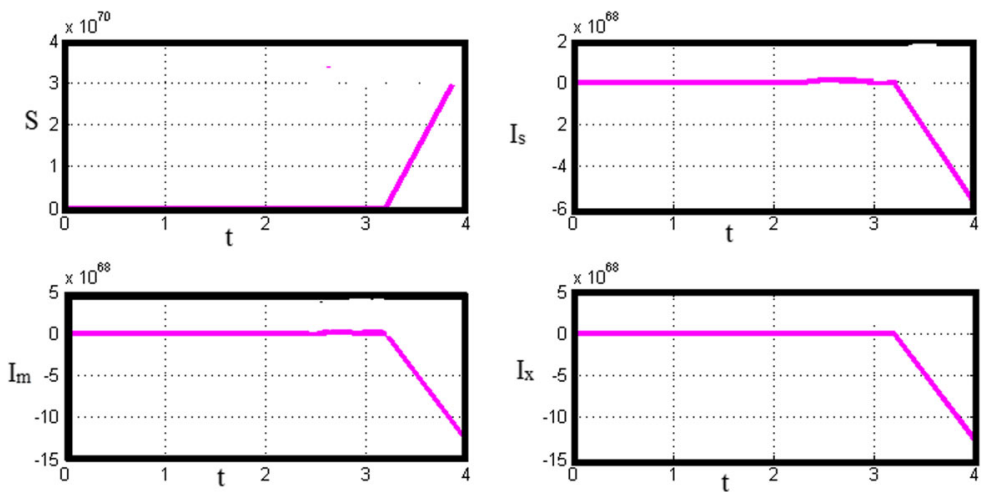

Fig. 10 The approximate solutions with $\theta=0.5, \xi=0.5, h=1$ by using TMM

in Fig. 4. Figure 5, shows the behavior of the approximate solutions for SDE model by using the proposed method and TMM in comparison with the result with deterministic model when $\xi=0$. We observe that there is excellent agreement with the solutions to the corresponding deterministic case and the solution of suggested technique in Fig. 6. Our method converges for large $h$ and preserves the positivity of the model state variables, as we can see in Figs. 7, 8, and 9. Figure 10 shows TMM is unstable for larger $h$ and the NTMM better than TMM where NTMM has large stability regions.

\section{Conclusions}

In this paper, we constructed NTMM to introduce numerically the approximate solution of a stochastic multi-strain TB model. The proposed method is based on choosing the weight factor $\theta$. The main advantage of this method is that it can be explicit or implicit with large stability regions as we see in our results. Special attention is given to study mean-square stability. Some numerical results are used to show the accuracy of the NTMM, and some figures are used to demonstrate how the solutions change when $\theta$ take different values. All computations in this paper are performed using MATLAB programming.

\section{Abbreviations}

DIMM: Drift implicit balanced Milstein method; EMM: Explict Milstein method; NDIMM: Non-standard drift implicit balanced Milstein method; NEMM: Non-standard explict Milstein method; NSFD: Non-standard finite difference; NTMM: A nonstandard theta Milstein method; ODEs: Ordinary differential equations; PDEs: Partial differential equations; SDE: Stochastic differential equation; SFDM: Standard finite difference method; TB: Tuberculosis; TMM: Theta Milstein method

\section{Acknowledgements}

The authors sincerely thank the editors, reviewers, and everyone who provide the advice, support, help, and useful comments. The product of this research paper would not be possible without all of them.

\section{Authors' contributions}

This is a joint paper. All the manuscript works are done in an equal percentage of participation. The author(s) read and approved the final manuscript.

Funding

Not applicable.

\section{Availability of data and materials}

The datasets generated and/or analyzed during the current study are available from the corresponding author on reasonable request. 
Author details

${ }^{1}$ Department of Mathematics, Faculty of Science, Cairo University, Giza, Egypt. ${ }^{2}$ Department of Mathematics, Faculty of Education, Sana'a University, Sana'a, Yemen.

Received: 3 August 2019 Accepted: 13 February 2020

Published online: 11 March 2020

\section{References}

1. Ding, Y., Xu, M., Hu, L.: Asymptotic behaviour and stability of a stochastic model for AIDS transmission. Appl. Math. Comput. 204, 99-108 (2008)

2. Dalal, N., Greenhalgh, D., Mao, X. R.: A stochastic model of AIDS and condom use. J. Math. Anal. Appl. 325, 36-53 (2007)

3. Jiang, D., Ji, C., Shi, N., Yu, J.: The long time behavior of DI SIR epidemic model with stochastic perturbation. J. Math Anal. Appl. 372, 162-180 (2010)

4. Jovanovic, M., Krstic, M.: Stochastically perturbed vector-borne disease models with direct transmission. Appl. Math. Model. 36, 5214-5228 (2012)

5. Lahrouz, A., Omari, L., Kiouach, D.: Global analysis of a deterministic and stochastic nonlinear SIRS epidemic mode nonlinear analysis. Model. Control. 16, 59-76 (2011)

6. Lu, Q.: Stability of an SIRS system with random perturbations. Phys. A. 388, 3677-3686 (2009)

7. Yang, J., Wang, X., Li, X.: Global stability of an HIV model with stochastic perturbation. Asian-Eur. J. Math. 4.2, 349-358 (2011)

8. Yu, J., Jiang, D., Shi, N.: Global stability of two-group SIR model with random perturbation. J. Math. Anal. Appl. $\mathbf{3 6 0}$ 235-244 (2009)

9. Cresson, J., Pierret, F.: Continuous versus discrete structures I-discrete embeddings and ordinary differential equations. math.NA (2014)

10. Mickens, R. E.: Nonstandard finite difference models of differential equations. World Scientific (1994)

11. Liu, Q., Jiang, D., Hayat, T., Alsaedi, A.: Dynamics of a stochastic tuberculosis model with antibiotic resistance. Chaos, Solitons Fractals. 109, 223-230 (2018)

12. Vyambwera, S. M., WitbooiA, P.: Stochastic TB model for a crowded environment. J. Appl. Math., 1-8 (2018)

13. El Fatini, M., Lahrouz, A., Pettersson, R., Settati, A., Taki, R.: Stochastic stability and instability of an epidemic model with relapse. Appl. Math. Comput. 316, 326-341 (2018)

14. Liu, Q., Jiang, D.: The dynamics of a stochastic vaccinated tuberculosis model with treatment. Phys. A: Stat. Mech. Appl. 527, 121-274 (2019). https://doi.org/10.1016/j.physa.2019.121274

15. Aparicio, J. P., Castillo-ćavez, C.: Mathematical modelling of tuberculosis epidemics. Math. Biosci. Eng. 6(2), 209-237 (2009)

16. Brooks-Pollock, E., Roberts, G. O., Keeling, M. J.: A dynamic model of bovine tuberculosis spread and control in Great Britain. Nat. (Lett.) 511, 228-231 (2014)

17. Castillo-ćavez, C., Feng, Z.: To treat or not to treat: the case of tuberculosis. J. Math. Biol. 35(6), 629-656 (1997)

18. Nyabadza, F., Winkler, D.: A simulation age-specific tuberculosis model for the cape town metropole. S. Afr. J. Sci. 109(9/10), 1-7 (2013)

19. Perez, A. M., Ward, M. P., Charmandarián, A., Ritacco, V.: Simulation model of within-herd transmission of bovine tuberculosis in argentine dairy herds. Prev. Vet. Med. 54(4), 72-361 (2002)

20. White, P. J., Garnett, G. P.: Mathematical modelling of the epidemiology of tuberculosis. In: Michael, E., Spear, R. C. (eds.) Modelling Parasite Transmission and Control, vol. 673, pp. 127-140, (2010)

21. Sweilam, N. H., AL-Mekhlafi, S. M.: On the optimal control for fractional multi-strain TB Model. Optim. Control Appl. Meth. Published online in Wiley Online Library (wileyonlinelibrary.com). 37(6), 1355-1374 (2016)

22. Mickens, R. E.: Dynamic consistency: a fundamental principle for constructing nonstandard finite difference schemes for differential equations. J. Differ. Equ. Appl. 11(7), 645-653 (2005)

23. Mickens, R. E.: Advances in the applications of nonstandard finite difference schemes. World Scientific (2005)

24. Sweilam, N. H., Soliman, I. A., Al-Mekhlafi, S. M.: Nonstandard finite difference method for solving the multi-strain TB model. J. Egypt. Math. Soc. 25(2), 129-138 (2017)

25. Belova, O. V., Chuluunbaatar, O., Kapralova, M. I., Sweilam, N. H.: The role of the bacterial mismatch repair system in SOS-induced mutagenesis: a theoretical background. J. Theor. Biol. 332, 30-41 (2013)

26. Cohen, T., Murray, M.: Modeling epidemics of multidrug-resistant M. tuberculosis of heterogeneous fitness. Nat. Med. 10(10), 1117-1121 (2004)

27. Dye, C., Garnett, G. P., Sleeman, K., Williams, B. G.: Prospects for worldwide tuberculosis control under the who dots strategy. Directly observed short-course therapy. Lancet. 352(9144), 1886-1891 (1998)

28. Sweilam, N. H., AL-Mekhlafi, S. M.: Comparative study for multi-strain tuberculosis (TB) model of fractional order. J. Appl. Math. Inf. Sci. 10(4), 1403-1413 (2016)

29. Gokdogan, A., Merdan, M., Yildirim, A.: A multistage differential transformation method for approximate solution of Hantavirus infection model. Commun. Nonlinear Sci. Numer. Simul. 17(1), 1-8 (2002)

30. Yildirim, A., Cherruault, Y.: Analytical approximate solution of a SIR epidemic model with constant vaccination strategy by homotopy perturbation method. Kybernetes. 38(9), 1566-1575 (2009)

31. Bulut, H., Kumar, D., Singh, J., Swroop, R., Baskonus, H. M.: Analytic study for a fractional model of HIV infection of CD4 ${ }^{+}$T lymphocyte cells. Math. Nat. Sci. 2, 33-43 (2018)

32. Sweilam, N. H., AL-Mekhlafi, S. M.: Numerical study for multi-strain tuberculosis (TB) model of variable-order fractional derivatives. J. Adv. Res. 7(2), 271-283 (2016)

33. Arino, J., Soliman, I. A.: A model for the spread of tuberculosis with drug-sensitive and emerging multidrug-resistant and extensively drug resistant strains. Mathematical and Computational Modelling. Wiley (2015)

34. Arnold, L.: Stochastic Differential Equations: Theory and Applications. Wiley, New York (1972)

35. Friedman, A.: Stochastic Differential Equations and Their Applications. Academic Press, New York (1976) 
36. Mao, X:: Stochastic Differential Equations and Applications. Horwood, Chichester (1997)

37. Li, X. Y., Mao, X. R.: Population dynamical behavior of non-autonomous Lotka-Volterra competitive system with random perturbation. Discret. Contin. Dyn. Syst. 24, 523-545 (2009)

38. Higham, D. J.: A-stability and stochastic mean-square stability. BIT. 40, 404-409 (2000)

39. Talay, D., Tubaro, L.: Expansion of the global error for numerical schemes solving stochastic differential equations. Stoch. Anal. Appl. 8, 483-509 (1990)

40. World Health Organization: Multidrug and extensively drug-resistant TB (M/XDR-TB): 2012 global report on surveillance and response. World Health Organization (2012)

\section{Publisher's Note}

Springer Nature remains neutral with regard to jurisdictional claims in published maps and institutional affiliations.

\section{Submit your manuscript to a SpringerOpen ${ }^{\circ}$} journal and benefit from:

- Convenient online submission

- Rigorous peer review

- Open access: articles freely available online

- High visibility within the field

- Retaining the copyright to your article

Submit your next manuscript at $\gg$ springeropen.com 